\title{
Proceedings
}

\section{Suppression of an Effect of Terrain Unevenness on Accuracy of Height Measurement in UAV with Integrated Ultrasound Altimeter during Landing ${ }^{\dagger}$}

\author{
Pavel Dycka ${ }^{1}$, Premysl Janu ${ }^{2, *}$ and Josef Bajer ${ }^{3}$ \\ 1 Department of Aviation Technology, University of Defence in Brno, Brno, Czechia; pavel.dycka@unob.cz \\ 2 Department of Communication Technologies, Electronic Warfare and Radiolocation, University of Defence \\ in Brno, Brno, Czechia \\ 3 Department of Aviation Technology, University of Defence in Brno, Brno, Czechia; josef.bajer@unob.cz \\ * Correspondence: premysl.janu@unob.cz \\ + Presented at the 7th Electronic Conference on Sensors and Applications, 15-30 November 2020; Available \\ online: https://ecsa-7.sciforum.net/.
}

Published: 15 November 2020

\begin{abstract}
The goal of this paper is to examine filtration possibilities of ultrasonically measured height of unmanned aerial vehicle (UAV) for the suppression of terrain unevenness. The article presents two basic methods of the filtration, namely moving average method and Kalman filter, and it carries out performance comparison of the two methods with simulated data. The comparison implies that the performance of the two methods depends on character of the observed terrain and also on the accuracy of the initial ultrasound measurements before filtration.
\end{abstract}

Keywords: ultrasonic sensor; moving average; Kalman filter; unmanned aerial vehicle; height measurement

\section{Introduction}

The goal of the paper is to compare two methods used for suppression of an effect of terrain unevenness on accuracy of height measurement in UAV with integrated ultrasound altimeter during landing. Secure landing is one of the main requirements of current UAV operation. When the UAV is controlled by an operator on direct visibility, the landing process is possible to control manually or alternatively to use auxiliary system that informs about current height. However recently, the development is focused on regimes without direct visibility between UAV and its operator or the autonomous and semiautonomous modes of UAV flight [1]. The course of the whole flight itself is possible to divide into three phases, take off, cruising and landing. Just autonomous landing is usually the most safety critical of these phases. For this reason it is crucial to continually measure the UAV height as accurately as possible and to monitor the landing area for potential obstacles [2]. The obstacle detection can be performed in various ways, today most often with on-board camera [3]. For height determination it is beneficial to use ultrasound. In this case it is necessary to compare requested measurement accuracy with actually achievable. Measurement accuracy is dependent on several factors caused by the altimeter itself and by outside conditions. Here it is possible to consider two essential effects. These are transmission environment properties and the character of the terrain from which the ultrasound signal is reflected during the height measurement. This article is focused on the ways to suppress the impact of terrain unevenness on measured height.

\section{Filtration Methods}


Two basic method were chosen for the terrain unevenness suppression. The first method is moving average method. Moving average is a simple filtration technique which uses current measured value together with fixed amount of previous measurements to compute the current estimate [4]. The equation for moving average computation of height has the following shape:

$$
h_{e}(t)=\left[h_{m}(t)+h_{m}(t-1)+\cdots+h_{m}(t-n)\right] /[n+1],
$$

where $h_{e}(t)$ is the height estimate at time $t, h_{m}(t)$ is a height measurement at time $t, h_{m}(t-1)$ is a height measurement at time $t-1, h_{m}(t-n)$ is a height measurement at time $t-n$ and $n$ is the amount of past measurements used for the computation. In simulation in this paper the number $n=9$.

The second method used in this paper is Kalman filter. Kalman filter is a recursive filter used to determine inner state of linear dynamic system from a series of noisy measurements [5]. In this paper, simple Kalman filter with 1-D motion model with constant velocity was used. The prediction state equations were used in this form:

$$
\begin{aligned}
& \mathrm{X}_{\mathrm{t} \mid \mathrm{t}-1}=\mathrm{A}_{\mathrm{t} X \mathrm{t}-1 \mid \mathrm{t}-1,}, \\
& P_{t \mid t-1}=A_{t} P_{t-1 \mid t-1} A_{t}^{T}+Q_{t},
\end{aligned}
$$

where $\mathrm{x}_{\mathrm{k} \mid \mathrm{k}-\mathrm{i}}$ is a state vector prediction at time $\mathrm{t}$ computed from state vector estimate from time $\mathrm{t}-1$ ( $\mathrm{xt}$ $1 \mid \mathrm{t}-1), \mathrm{P}_{\mathrm{t} \mid \mathrm{t}-1}$ is a covariance matrix prediction at time $\mathrm{t}$ computed from covariance matrix of state at time $\mathrm{t}-1\left(\mathrm{P}_{\mathrm{t}-1 \mid \mathrm{t}-1}\right), \mathrm{Q}_{\mathrm{t}}$ is a process noise covariation matrix and $\mathrm{A}_{\mathrm{t}}$ is a 2-by-2 transformation matrix defined as:

$$
\mathrm{A}_{\mathrm{t}}=[1 \Delta \mathrm{t}, 01],
$$

where $\Delta t$ is sampling period. The specific motion model was chosen based on assumptions of constant altitude flight of the UAV and non-constant terrain profile [6].

\section{Simulation Scenarios}

The simulations were performed using to flight scenarios. In both of these scenarios, linear movement of a UAV was simulated in constant altitude. The terrain profile was non-constant in both scenarios which with constant altitude results in changing measured height during the simulations. The height measurement was simulate to occur for $100 \mathrm{~s}$ with 10 measurements per second being performed. Velocity of the UAV was set as $0.25 \mathrm{~m} / \mathrm{s}$, which results in total distance of $25 \mathrm{~m}$.

The terrain profile in the first scenario had three sections with constant height $(5 \mathrm{~m}, 7 \mathrm{~m}$ and again $5 \mathrm{~m}$ ) and two sections of linear height change set between the constant sections. The flight above each of the sections took $20 \mathrm{~s}$. The second scenario used terrain profile with fluently changing height and was down sloped for the most of its duration. The simulated height was between 4.8 and $6.1 \mathrm{~m}$ in this scenario. Two variants of terrain unevenness were also applied in both of the scenarios. The small unevenness uses maximal difference from the original terrain of $0.05 \mathrm{~m}$ and the large unevenness uses maximal difference from the original terrain of $0.15 \mathrm{~m}$. All of the resulting terrain situations are presented in Figure 1. The figure uses negative height to illustrate the actual terrain character as would be visible by outside observer. The same is true for all following figures.

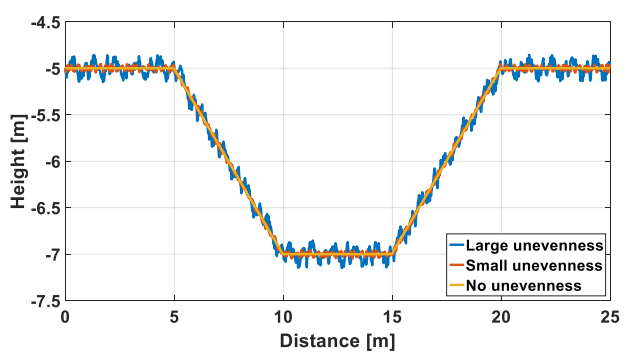

(a)

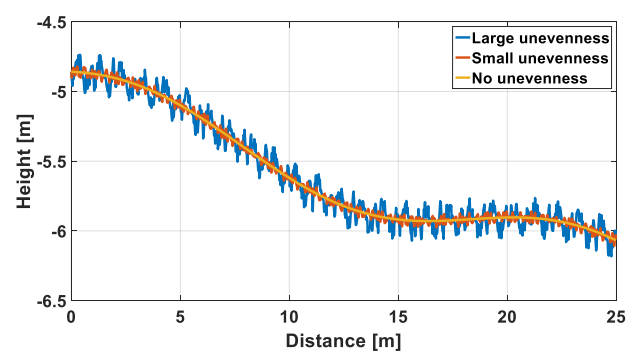

(b)

Figure 1. Depiction of (a) Scenario 1 and (b) Scenario 2, both including unevenness's. 
Apart from that, two versions of measurement noise were also applied on all the terrain situations. The first version consisted of Gaussian noise with standard deviation of $50 \mathrm{~mm}$, which simulates ultrasonic measurement with threshold detector. The second version also consisted of Gaussian noise, this time with standard deviation of $10 \mathrm{~mm}$, which simulates ultrasonic measurement with intra-pulse modulation technique [7].

\section{Evaluation of Simulation Results}

The simulations were performed according to the scenarios mentioned above using both filtration algorithms. Six series of waveforms were plotted and individual algorithms were analyzed. Figure 2 shows curves of the profile mapping of the surface 1 for no unevenness. In Figure $2 \mathrm{a}$ maximum error of Kalman filter is $82 \mathrm{~mm}$ and maximum error of moving average is $77 \mathrm{~mm}$ and in Figure $2 \mathrm{~b}$ maximum error of Kalman filter is $46 \mathrm{~mm}$ and maximum error of moving average is 50 $\mathrm{mm}$.

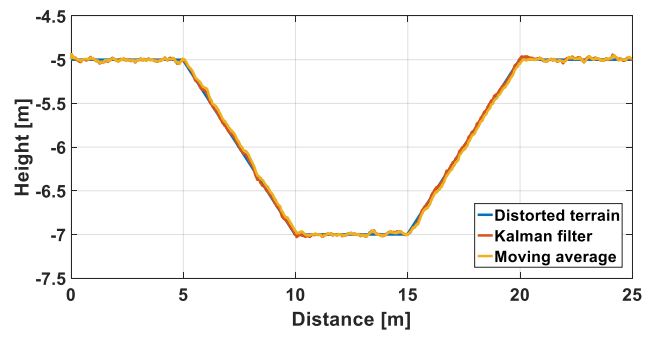

(a)

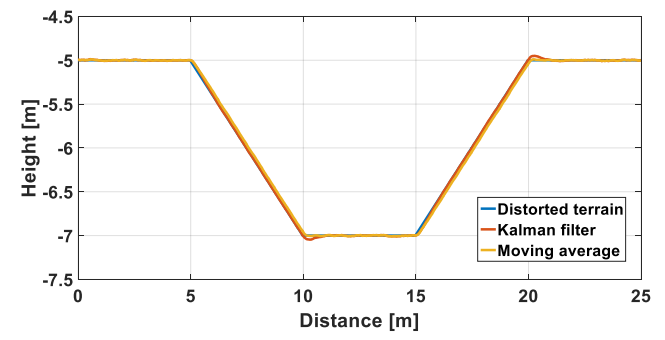

(b)

Figure 2. Simulation results of surface approximation by Kalman filter and moving average algorithm for defined surface profile 1 with no unevenness: (a) using threshold detector; (b) using intra-pulse phase signal modulation.

The blue curve represents the real surface profile, the yellow curve represents the use of moving average algorithm for its approximation, and the red curve shows the implementation of Kalman filter. As is seen from the figures, using intra-pulse phase modulation and moving average algorithm, very accurate approximations of the defined surface profile is possible to obtain, the yellow and blue curves are almost identical. When using the Kalman filter algorithm, undesirable overshoots occur in two areas. If the threshold detector is used, the overshoots with the Kalman filter algorithm is possible to see again. The implementation of the moving average again ensures better mapping of the defined surface profile. Figure 3 shows curves of the profile mapping of the surface 1 for small unevenness. In Figure 3a maximum error of Kalman filter is $85 \mathrm{~mm}$ and maximum error of moving average is $68 \mathrm{~mm}$ and in Figure 3b maximum error of Kalman filter is $51 \mathrm{~mm}$ and maximum error of moving average is $65 \mathrm{~mm}$.

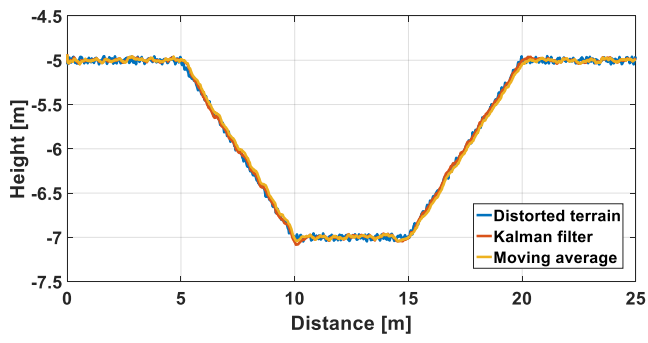

(a)

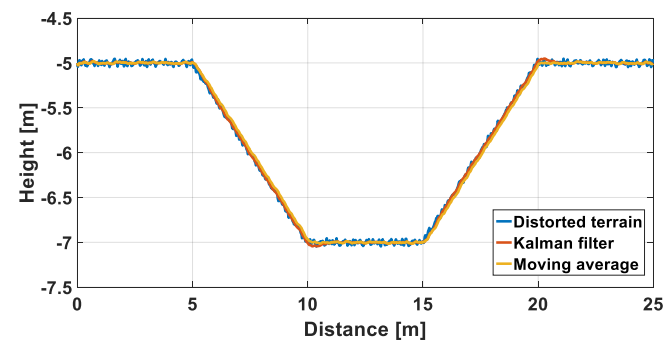

(b)

Figure 3. Simulation results of surface approximation by Kalman filter and moving average algorithm for defined surface profile 1 with small unevenness: (a) using threshold detector; (b) using intra-pulse phase signal modulation. 
In this case, it is again possible to conclude that the implementation of the moving average algorithm more accurately approximates shape of the defined surface. The application of the Kalman filter algorithm here again represents undesirable overshoots in the above-mentioned two areas. Figure 4 shows curves of the profile mapping of the surface 1 for large unevenness. In Figure $4 \mathrm{a}$ maximum error of Kalman filter is $110 \mathrm{~mm}$ and maximum error of moving average is $140 \mathrm{~mm}$ and in Figure $4 \mathrm{~b}$ maximum error of Kalman filter is $82 \mathrm{~mm}$ and maximum error of moving average is 133 $\mathrm{mm}$.

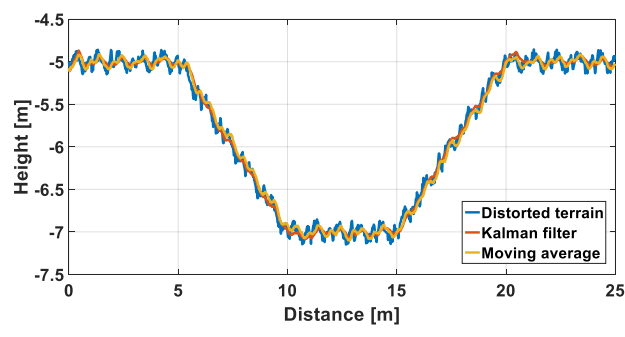

(a)

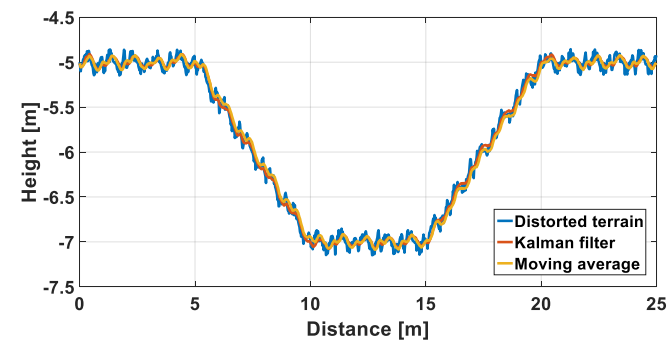

(b)

Figure 4. Simulation results of surface approximation by Kalman filter and moving average algorithm for defined surface profile 1 with large unevenness: (a) using threshold detector; (b) using intra-pulse phase signal modulation.

In this case, it is possible to conclude from the waveforms that the intra-pulse phase signal modulation does not significantly affect the mapping quality of the defined surface. The curves are similar. From the comparison of moving average algorithm and the Kalman filter implementation, it is also possible to conclude that the method of approximation of the defined surface does not differ significantly. Figure 5 shows curves of the profile mapping of the surface 2 for no unevenness. In Figure 5a maximum error of Kalman filter is $54 \mathrm{~mm}$ and maximum error of moving average is $53 \mathrm{~mm}$ and in Figure $5 \mathrm{~b}$ maximum error of Kalman filter is $11 \mathrm{~mm}$ and maximum error of moving average is $9 \mathrm{~mm}$.

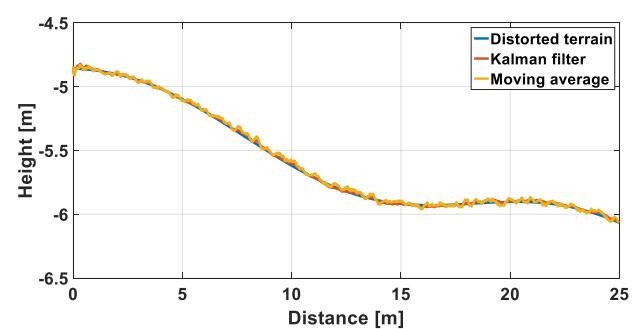

(a)

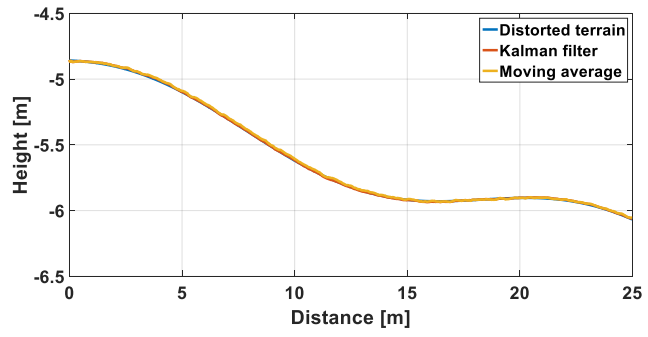

(b)

Figure 5. Simulation results of surface approximation by Kalman filter and moving average algorithm for defined surface profile 2 with no unevenness: (a) using threshold detector; (b) using intra-pulse phase signal modulation.

It is possible to see from the graphs that in the case of using the threshold detector and the profile without step changes, the implementation of the Kalman filter will ensure a better approximation of the surface. Thus, this algorithm here reduces the need to use intra-pulse phase modulation. In the case of the moving average, there is more noise. In the case of the application of the intra-pulse phase modulation, all curves are significantly similar, so it is not possible to unambiguously give priority to the use of one specific algorithm. Figure 6 shows curves of the profile mapping of the surface 2 for small unevenness. In Figure 6a maximum error of Kalman filter is $40 \mathrm{~mm}$ and maximum error of moving average is $49 \mathrm{~mm}$ and in Figure $6 \mathrm{~b}$ maximum error of Kalman filter is $25 \mathrm{~mm}$ and maximum error of moving average is $19 \mathrm{~mm}$. 


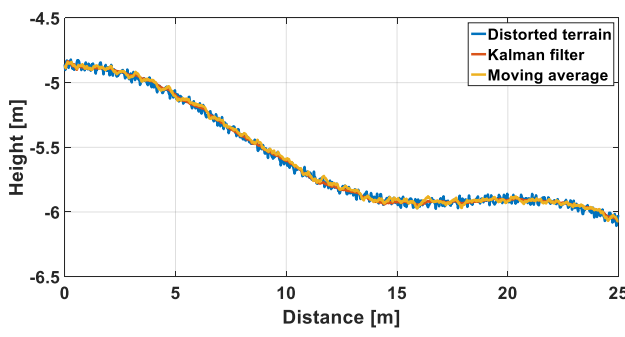

(a)

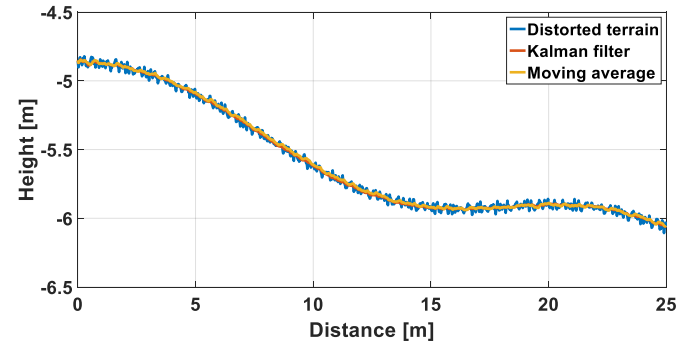

(b)

Figure 6. Simulation results of surface approximation by Kalman filter and moving average algorithm for defined surface profile 2 with small unevenness: (a) using threshold detector; (b) using intra-pulse phase signal modulation.

In this case, again using the threshold detector, the Kalman filter algorithm shows more accurate approximation of the given surface profile. Here again, it would be possible to disable intra-pulse phase signal modulation. In the case of the use of the intra-pulse phase modulation, no significant difference between two mentioned algorithms is observed. Figure 7 shows curves of the profile mapping of the surface 2 for large unevenness. In Figure 7a maximum error of Kalman filter is 87 $\mathrm{mm}$ and maximum error of moving average is $111 \mathrm{~mm}$ and in Figure $7 \mathrm{~b}$ maximum error of Kalman filter is $79 \mathrm{~mm}$ and maximum error of moving average is $92 \mathrm{~mm}$.

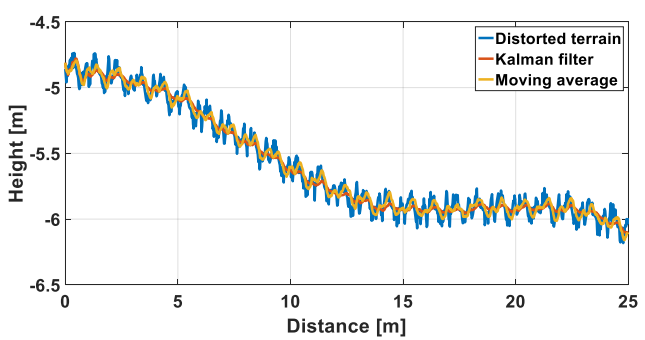

(a)

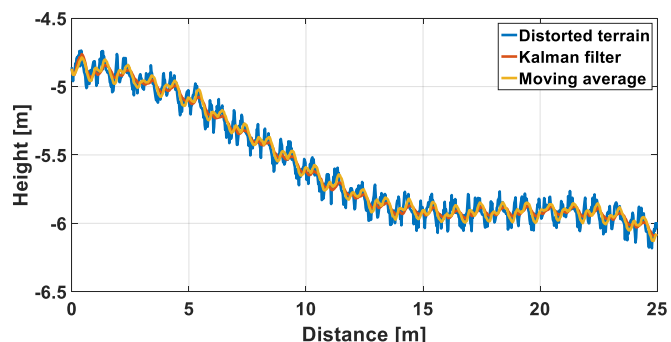

(b)

Figure 7. Simulation results of surface approximation by Kalman filter and moving average algorithm for defined surface profile 2 with large unevenness: (a) using threshold detector; (b) using intra-pulse phase signal modulation.

In the case of the large unevenness of the defined surface, here, it is not possible to recognize significant difference between the simulation waveforms using the threshold detector and the signal with intra-pulse phase modulation. The curves are considerably similar. Also, the implementation of the moving average algorithm or the Kalman filter cannot be unambiguously preferred. In this case, the algorithm with lower computational complexity is possible to use.

\section{Conclusion}

The paper was devoted to the analysis of two methods for the elimination of surface unevenness when measuring height using ultrasound in the landing phase of an unmanned aerial vehicle. These methods were the moving average and the Kalman filter. One particular algorithm cannot be clearly preferred. Each algorithm has its specifics for particular surface profile and for different types of its unevenness's, which is possible to evaluate from the performed simulations. For a step-changing surface profile and very small unevenness's, the moving average shows better results. The Kalman filter algorithm represents more different values at the moment of the step change of the profile. In the case of large unevenness's, the waveforms represent similar character. It is possible to use the algorithm with lower computational complexity. In the case of continuous changes of the profile of given surface and very small unevenness's, the Kalman filter algorithm is possible to prefer when exciting the ultrasonic transducer with a signal without intra-pulse modulation. It eliminates surface unevenness's significantly better. This could be confusing according to the values of maximal errors. 
Here the errors are calculated in relation to the ideal surface profile. So, in the case of moving average, there is a delay, especially in the first scenario. Therefore, the character of the curve seems to be better, but the values of the maximum errors show a discrepancy in some cases.

The aim of further work in this area is to define more surface profiles with different unevenness's, to perform a simulation with the implementation of the mentioned algorithms and to analyse these results. This will be followed by experimental verification of height measurements with specific transducers on an unmanned aerial vehicle, and thus verification of the results of simulations in practice.

Acknowledgments: The work presented in this paper has been supported by the Czech Republic Ministry of Defence-University of Defence development program "Research of sensor and control systems to achieve battlefield information superiority" and by the Czech Republic Ministry of Education, Youth and Sports University of Defence student research program "Implementation of modern technologies in avionic systems".

\section{References}

1. Błachuta, M.; Czyba, R.; Janusz, W.; Szafrański, G. Data fusion algorithm for the altitude and vertical speed estimation of the VTOL platform. J. Intell. Robot. Syst. Theory Appl. 2014, doi:10.1007/s10846-013-9917-4.

2. Shijith, N.; Dharmana, M.M. Sonar Based Terrain Estimation \& Automatic Landing of Swarm Quadrotors. In Proceedings of the IEEE International Conference on Circuit, Power and Computing Technologies, ICCPCT, Kollam, India, 20-21 April 2017.

3. Janusz, W.; Mielimaka, R.; Niezabitowski, M.; Orwat, J.; Sikora, P. Surface monitoring of water basins based on use of autonomous flying robots. In Proceedings of the 2015 20th International Conference on Methods and Models in Automation and Robotics, MMAR, Miedzyzdroje, Poland, 24-27 August 2015.

4. Liu, S.T. Nonlinear Signal Processing: A Statistical Approach. Technometrics 2006, doi:10.1198/tech.2006.s355.

5. Strid, I.; Walentin, K. Block Kalman filtering for large-scale DSGE models. Comput. Econ. 2009, doi:10.1007/s10614-008-9160-4.

6. Musoff, H; Zarchan, P. Fundamentals of Kalman Filtering: A Practical Approach, 4th ed.; American Institute of Aeronautics and Astronautics: 2015;

7. Dycka, P.; Janu, P.; Bajer, J.; Bystricky, R. Phase-Coded Modulation Based Time-of-Flight Measurement Improvement for Piezoelectric Ceramic Transducers. IEEE Trans. Ultrason. Ferroelectr. Freq. Control 2020, $1-$ 1, doi:10.1109/TUFFC.2020.3029329.

(C) 2020 by the authors. Submitted for possible open access publication under the terms and conditions of the Creative Commons Attribution (CC BY) license (http://creativecommons.org/licenses/by/4.0/). 